\title{
Odd and Even Modes of Neutron Spin Resonance in the Bilayer Iron-Based Superconductor $\mathrm{CaKFe}_{4} \mathrm{As}_{4}$
}

\author{
Tao Xie, ${ }^{1,2}$ Yuan Wei, ${ }^{1,2}$ Dongliang Gong, ${ }^{1,2}$ Tom Fennell, ${ }^{3}$ Uwe Stuhr, ${ }^{3}$ Ryoichi Kajimoto, ${ }^{4}$ Kazuhiko Ikeuchi, \\ Shiliang $\mathrm{Li}^{1,2,6}$ Jiangping $\mathrm{Hu},{ }^{1,2,6}$ and Huiqian $\mathrm{Luo}^{1, *}$ \\ ${ }^{1}$ Beijing National Laboratory for Condensed Matter Physics, Institute of Physics, Chinese Academy of Sciences, \\ Beijing 100190, China \\ ${ }^{2}$ University of Chinese Academy of Sciences, Beijing 100049, China \\ ${ }^{3}$ Laboratory for Neutron Scattering and Imaging, Paul Scherrer Institute, CH-5232 Villigen, Switzerland \\ ${ }^{4}$ Materials and Life Science Division, J-PARC Center, Japan Atomic Energy Agency, Tokai, Ibaraki 319-1195, Japan \\ ${ }^{5}$ Neutron Science and Technology Center, Comprehensive Research Organization for Science and Society, \\ Tokai, Ibaraki 319-1106, Japan \\ ${ }^{6}$ Collaborative Innovation Center of Quantum Matter, Beijing 100190, China
}

(Received 7 February 2018; published 29 June 2018)

\begin{abstract}
We report an inelastic neutron scattering study on the spin resonance in the bilayer iron-based superconductor $\mathrm{CaKFe}_{4} \mathrm{As}_{4}$. In contrast to its quasi-two-dimensional electron structure, three strongly $L$ dependent modes of spin resonance are found below $T_{c}=35 \mathrm{~K}$. The mode energies are below and linearly scale with the total superconducting gaps summed on the nesting hole and electron pockets, essentially in agreement with the results in cuprate and heavy fermion superconductors. This observation supports the sign-reversed Cooper pairing mechanism under multiple pairing channels and resolves the long-standing puzzles concerning the broadening and dispersive spin resonance peak in iron pnictides. More importantly, the triple resonant modes can be classified into odd and even symmetries with respect to the distance of Fe-Fe planes within the Fe-As bilayer unit. Thus, our results closely resemble those in the bilayer cuprates with nondegenerate spin excitations, suggesting that these two high- $T_{c}$ superconducting families share a common nature.
\end{abstract}

DOI: 10.1103/PhysRevLett.120.267003

Understanding the superconducting mechanism in unconventional superconductors, such as copper oxides, heavy fermions, iron pnictides, and iron chalcogenides, is one of the most important topics in modern condensed matter physics [1-3]. On cooling below the superconducting transition temperature $\left(T_{c}\right)$ in these materials, the spin excitations form a resonant peak with enhanced susceptibility at a certain energy and around the antiferromagnetic (AFM) wave vector of the parent compounds. This socalled neutron spin resonance, which is argued to be a spin-1 collective mode of particle-hole excitations in the superconducting state, gives prominent evidence for the magnetic Cooper pairing in cuprates and heavy fermion superconductors [3-7].

The multiband physics from $\mathrm{Fe} 3 d$ orbitals in iron-based superconductors opens a new opportunity to explore the unconventional superconductivity [8,9]. In iron pnictides or chalcogenides, the sign-reversed $s$-wave $\left(s_{ \pm}\right)$Cooper pairing can be obtained in both weak coupling approaches [10-13] and strong correlated electron models [14] and has been supported by many experimental evidences [15-19]. In the $s_{ \pm}$superconducting state, a spin resonance is theoretically predicted to arise at the wave vector $\mathbf{Q}$ linking between hole-electron or electron-electron pockets, which is experimentally observed in many systems [20-40]. If the resonance is indeed a spin exciton in the superconducting state, it should be a sharp peak bound at an energy $\left(E_{R}\right)$ below the pair breaking energy, namely, the total superconducting gap summed on the two pockets linked by Q: $\Delta_{\text {tot }}=\left|\Delta_{k}\right|+\left|\Delta_{k+Q}\right|[11,12,34,41]$. Here, $\Delta_{k}$ and $\Delta_{k+Q}$ have opposite signs (probably different values) to yield a finite coherence factor of this process, enhanced by the interband Fermi surface nesting under intraorbital Coulomb repulsion $[12,13]$. In contrast, a nonresonant broad peak in the magnetic spectrum below $T_{c}$ is expected above twice the superconducting gap $(2 \Delta)$ in the sign-preserved $\left(s_{++}\right)$state [42-44]. However, compared to the resonance mode observed in copper oxides [5,6], spin resonances in iron pnictides are actually much broader in energy distribution and more dispersive both in plane and along the $L$ direction [29-33]. The lack of sharpness in both energy and momentum spaces may be attributed to the complex multiorbital nature in iron-based superconductors that can lead to multiple resonant modes and spin anisotropy [45-49].

To further understand the spin resonance in iron-based superconductors, it is essential to make a full comparison to all behaviors of the resonant mode observed in cuprates. In the hole-doped bilayer $\mathrm{YBa}_{2} \mathrm{Cu}_{3} \mathrm{O}_{6+\delta}(\mathrm{YBCO})$ system, 
the spin resonance exhibits distinguished odd and even $L$ symmetries due to the nondegenerate interlayer magnetic excitations [5,50,51], which is later confirmed in another bilayer system $\mathrm{Bi}_{2} \mathrm{Sr}_{2} \mathrm{CaCu}_{2} \mathrm{O}_{8+\delta}$ (Bi2212) [6,52]. These two different modes of spin resonance evolve in a strikingly similar doping dependence in both systems, and their separation in energy is fully determined by a weak AFM interaction between $\mathrm{Cu}-\mathrm{O}$ planes within the bilayer unit, giving strong evidence for the magnetically mediated superconducting pairing mechanisms. However, this even mode has never been observed in iron-based superconductors, which seems to suggest that the spin resonance may have different origins in these two high- $T_{c}$ families.

In this Letter, we report an inelastic neutron scattering study on the spin excitations of stoichiometric iron-based superconductor $\mathrm{CaKFe}_{4} \mathrm{As}_{4}$ (1144 compound) with Fe-As bilayer structure [Fig. 1(a)]. Three spin resonance modes are identified at the wave vector $\mathbf{Q}$ from $\Gamma$ to $M$ point [Fig. 1(b)], where the resonance energies and the mode intensities are directly proportional to the total superconducting gaps summed on the nesting electron and hole bands. In contrast to its quasi-two-dimensional (2D) electron structure, the resonance intensity for all three modes is highly $L$ dependent with two opposite harmonic modulations, showing either odd symmetry $\sim|F(Q)|^{2} \sin ^{2}(z \pi L)$ or even symmetry $\sim|F(Q)|^{2} \cos ^{2}(z \pi L)$ [Figs. 1(c)-1(f)]. Here, $F(Q)$ is the magnetic form factor of $\mathrm{Fe}^{2+}$, and $z c=5.855 \AA$ $(z=0.4636)$ is the distance between adjacent Fe-Fe planes within the Fe-As bilayer unit [Fig. 1(a)]. We argue that such phenomenon is essentially similar to the nondegenerate bilayer magnetic excitations in YBCO [5] but under multiband pairing mechanism [8].

We prepared high-quality single crystals of $\mathrm{CaKFe}_{4} \mathrm{As}_{4}$ using the self-flux method according to the previous reports
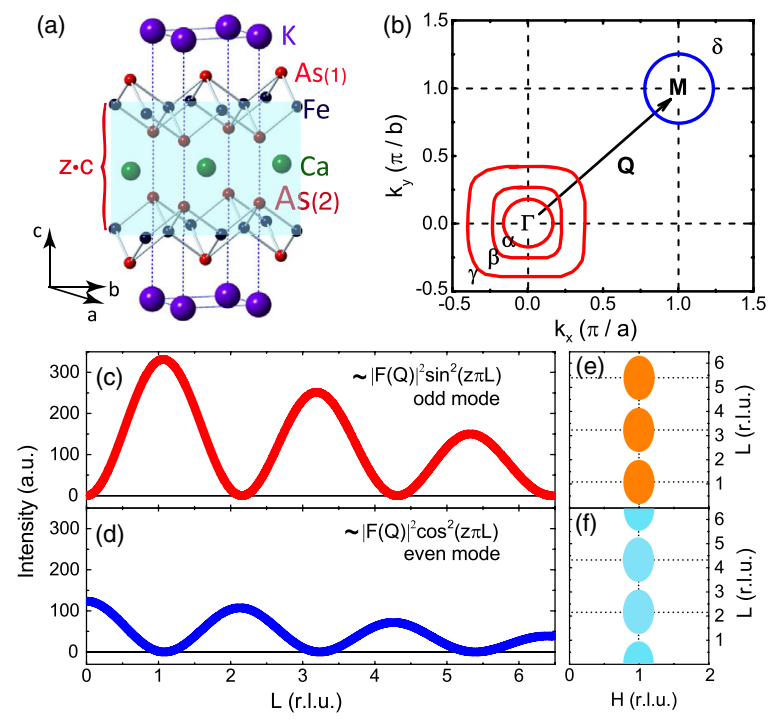

FIG. 1. (a) Crystal structure of $\mathrm{CaKFe}_{4} \mathrm{As}_{4}$. (b) 2D Fermi surfaces with nesting wave vector $\mathbf{Q}$ from $\Gamma$ to $M$ point. (c)-(f) Odd and even $L$ symmetries of the spin resonance.
[53-56]. X-ray diffraction, resistivity, and magnetization measurements suggest our crystals have a very homogenous quality with sharp superconducting transitions around $35 \mathrm{~K}$, where the potential impurity phases from $\mathrm{CaFe}_{2} \mathrm{As}_{2}$ or $\mathrm{KFe}_{2} \mathrm{As}_{2}$ (122 compound) are completely absent. Neutron scattering experiments were carried out using thermal triple-axis spectrometer EIGER at SINQ, PSI, Switzerland, with a fixed final energy $E_{f}=14.7 \mathrm{meV}$ and about $2 \mathrm{~g}$ ( 200 pieces) of coaligned crystals [64]. Time-of-flight (TOF) neutron scattering experiments were carried out at 4SEASONS spectrometer (BL-01) at J-PARC, Tokai, Japan, with incident energy $E_{i}=42$ and $23 \mathrm{meV}, k_{i}$ parallel to the $c$ axis, chopper frequency $f=250 \mathrm{~Hz}$, and a total sample mass of about $4.3 \mathrm{~g}$ ( $\sim 00$ pieces) [65-67]. The scattering plane was $[H, 0,0] \times[0,0, L]$, defined using the magnetic unit cell with 2-Fe atoms similar to that of the 122 parent compounds: $a_{M}=b_{M}=5.45 \AA, c=12.63 \AA$, in which the wave vector $\mathbf{Q}$ at $\left(q_{x}, q_{y}, q_{z}\right)$ is $(H, K, L)=\left(q_{x} a_{M} / 2 \pi\right.$, $\left.q_{y} b_{M} / 2 \pi, q_{z} c / 2 \pi\right)$ reciprocal lattice units (r.l.u.). The collinear ( $C$-type) AFM order similar to $\mathrm{CaFe}_{2} \mathrm{As}_{2}$ (or the noncollinear spin vortex phase [68]), which is expected to form magnetic Bragg peaks at $Q=(1,0, L)$ [and $Q=(0,1, L)](L= \pm 1, \pm 3, \pm 5, \ldots)$, does not exist based on our neutron diffraction experiments (Supplemental Material [56]). Even so, the spin excitations still emerge around $Q=(1,0)$, coinciding with the Fermi surface nesting vector from $\Gamma$ to $M$ point [Fig. 1(b)], similar to many other iron pnictides [Figs. 1(e) and 1(f)] [2,3].

Figure 2 gives the key results of this paper. After subtracting the intensity of spin excitations in the normal state $(T=40 \mathrm{~K})$ from $E=2$ to $22 \mathrm{meV}$ at $Q=(1,0, L)$ with $L$ in the range $2-3$, we can identify three spin resonance peaks in the superconducting state $(T=1.5 \mathrm{~K})$ at $E_{R}=9.5 \pm 0.5,13 \pm 0.5,18.3 \pm 0.5 \mathrm{meV}$, respectively [Fig. 2(a)]. The intensity distribution of all three peaks separates into two groups, as clearly shown by the TOF neutron experiments [Fig. 2(b)]. Although the 9.5 and $13 \mathrm{meV}$ modes overlap with each other, it seems all three modes are energy resolution limited and nearly nondispersive along both the $K$ and $L$ directions. The temperature dependence of all three modes confirms their coupling to superconductivity: the intensity gain decreases like a superconducting order parameter, which ceases at $T_{c}=$ $35 \mathrm{~K}$ [Fig. 2(c)]. A spin gapped feature with intensity loss below $T_{c}$ is also found below $E=7 \mathrm{meV}$. More interestingly, all three modes show strong but different $L$ dependences with the maximums at $L=3$ for the former two modes and $L=2$ for the latter one [Fig. 2(a)]. Thus, we have further measured the spin excitations over a large range of $Q=(1,0, L)$ with $L=0-6$, where those below $L=2$ cannot be measured for $E \geq 16 \mathrm{meV}$ due to the scattering restriction. The results are summarized in Figs. 2(d)-2(f). Obviously, two opposite symmetries along $L$ can be identified for maximum around $L=$ odd or even, 

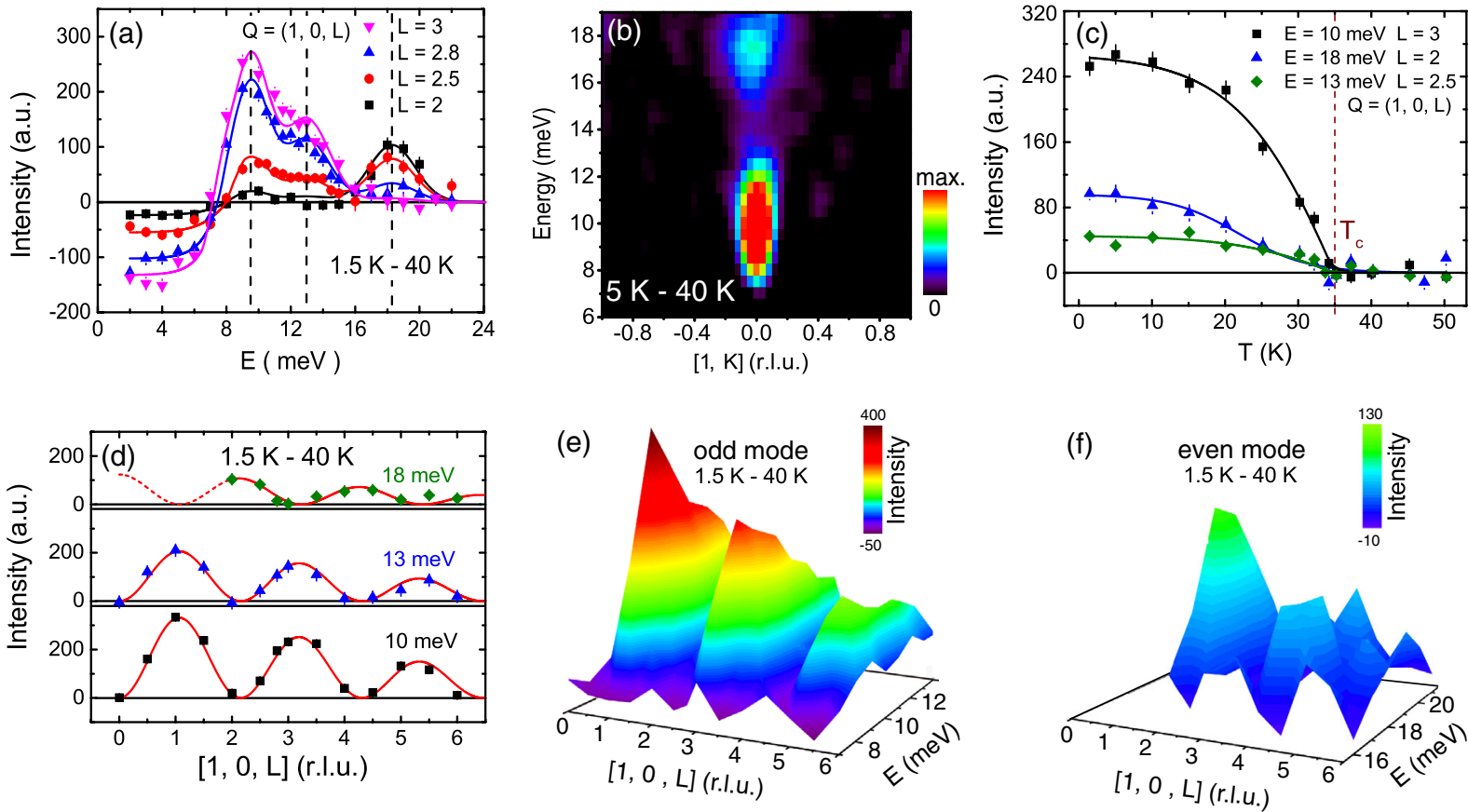

FIG. 2. (a) Energy dependence of the spin resonances at $Q=(1,0, L)$. The solid lines are guides to eyes. (b) $2 \mathrm{D}$ slice in $E$ vs $K$ of the spin resonances. (c) Temperature dependence and (d) $L$ dependence of three resonance modes at $E=10,13,18 \mathrm{meV}$. The red solid and dashed lines are fitting results by $|F(Q)|^{2} \sin ^{2}(z \pi L)$ [or $|F(Q)|^{2} \cos ^{2}(z \pi L)$ ] function. (e),(f) $L$ modulation of the odd resonance modes and the even resonance mode at different energy ranges.

much similar to the cases in bilayer cuprates $\mathrm{YBCO}$ and Bi2212 [5,6,50-52]. In the metallic YBCO, both odd and even modes of spin resonance are found corresponding to the acoustic and optical spin waves in the AFM insulating phase [69]. Although there is no evidence for any optical branch from antiphase spin excitations in the paramagnetic $\mathrm{CaKFe}_{4} \mathrm{As}_{4}$, by simply considering the symmetric and antisymmetric combinations from the contribution of magnetically decoupled $\mathrm{Fe}$-As bilayers similar to metallic YBCO [56,70], we can obtain both odd and even $L$ symmetries of the spin excitations. Here, the intensity of two spin resonances at $E_{R}=9.5$ and $13 \mathrm{meV}$ follows the $L$ modulation $|F(Q)|^{2} \sin ^{2}(z \pi L)$ (so-called odd mode), and the one at high energy $\left(E_{R}=18.3 \mathrm{meV}\right)$ can be described by $|F(Q)|^{2} \cos ^{2}(z \pi L)$ (so-called even mode) instead, with respect to the distance $(z c)$ between the adjacent $\mathrm{Fe}-\mathrm{Fe}$ planes within the Fe-As bilayer unit [Fig. 1(a)] [56]. The data points agree very well with such sine-squared (cosinesquared) modulation, as shown in Fig. 2(d). Here, we have $z=0.4636$ for the unique bilayer structure due to the shift of the intermediate FeAs layer out of their high-symmetry positions $(z=0.5)$ [53-55]. Consequently, the peak positions actually shift to nonintegral $L$ in comparison with the high-symmetric structure, such as $L=1.08,3.24$, and 5.39 (odd mode) or $L=2.16,4.31$, and 6.47 (even mode), etc., [Figs. 1(c)-1(f)]. Unlike the weak $L$ modulation of spin resonance in 122-type iron pnictides [29-31], the minimum intensity at each valley here is near zero [56].
Figure 3 summarizes the intensity distribution of the spin resonances and spin gap within $[H, K]$ plane. All three resonance modes and the spin gap follow Gaussian line shapes around $Q=(1,0, L)$. While both the intensity loss at $3 \mathrm{meV}$ and the intensity gain at $10 \mathrm{meV}$ look like ellipses

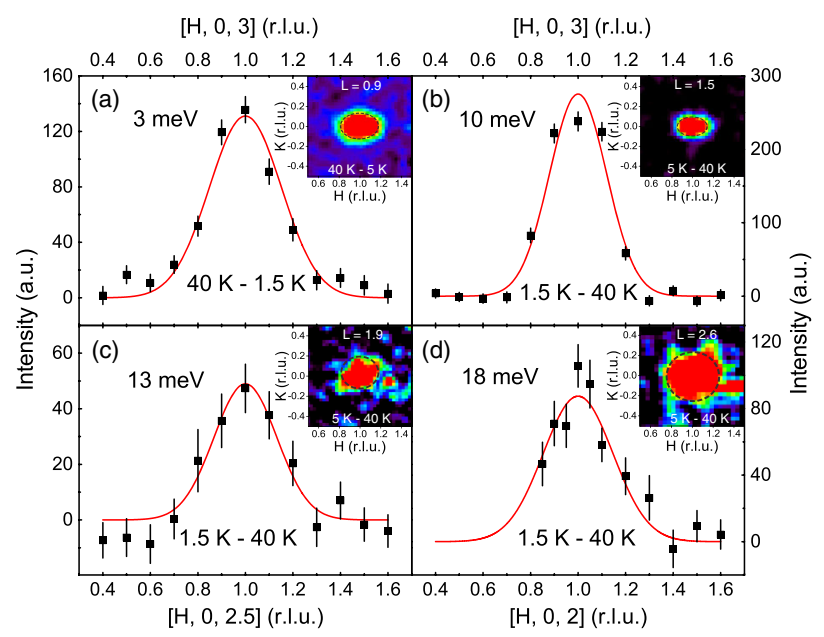

FIG. 3. Constant-energy scans along the $H$ direction for (a) the spin gap at $3 \mathrm{meV}$ and (b)-(d) three resonance modes at 10,13 , $18 \mathrm{meV}$ with intensity differences below and above $T_{c}$. The red solid lines are Gaussian fits to the data. (Insets) 2D slices at half maximum of the intensity with identical energy transfer but different Ls [56]. 
elongated along the $H$ direction, similar to the hole-doped $\mathrm{Ba}_{1-x} \mathrm{~K}_{x} \mathrm{Fe}_{2} \mathrm{As}_{2}$ [30], the 13 and $18 \mathrm{meV}$ resonant modes are more like circles in the $[H, K]$ plane. The peak width at half maximum of the intensity is determined by the dispersion of the paramagnetic excitation, and the relative intensity depends on the energy transfer coupled with the $L$ position in the TOF neutron scattering experiment when $k_{i} \| c$.

The triple modes of spin resonance in $\mathrm{CaKFe}_{4} \mathrm{As}_{4}$ can be naturally explained by multiple pairing channels. Although the density functional theory calculations predict ten Fermi pockets (six hole bands and four electron bands) [71], the angle-resolved-photoemission-spectroscopy measurements reveal three hole pockets $(\alpha, \beta, \gamma)$ around the $\Gamma$ point and one electron pocket $(\delta)$ around the $M$ point, with large diversity of the superconducting gaps and 2D shapes of each pocket [Fig. 1(b)] [72]. The observation of several full gaps and matched sizes of electron and hole pockets is consistent with the $s_{ \pm}$pairing scenario under Fermi surface nesting. Thus, three different values of the total superconducting gaps $\left(\Delta_{\text {tot }}\right)$ summed on the nesting hole and electron pockets should yield three modes of spin resonance at different energies [56]. It turns out that the resonance energy and the maximum intensity gain for each mode $[\Delta S(Q, \omega)]$ show contrary linear dependence with $\Delta_{\text {tot }}[56,72]$, as shown in Figs. 4(a) and 4(b). In fact, a universal relationship $E_{R} / 2 \Delta=0.64$ was proposed among copper oxide, heavy fermion, and iron pnictide superconductors [7], where $2 \Delta$ is twice the superconducting gap in the single band case. We then summarize the reported results about $E_{R}$ and $\Delta_{\text {tot }}$ in Fig. 4(d) for ironbased superconductors [21-31,44,72-86]. The same linear scaling with $E_{R}=0.64 \Delta_{\text {tot }}$ can also describe these data
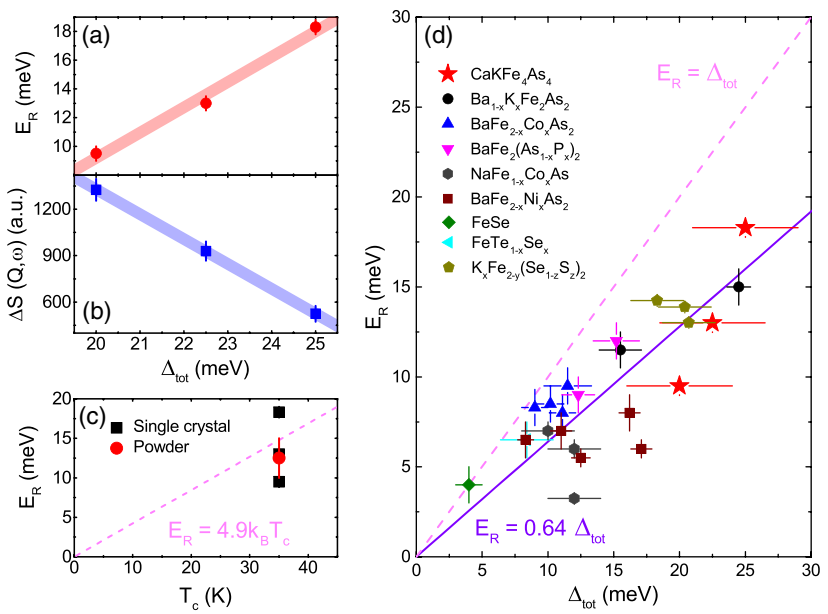

FIG. 4. (a),(b) The linear relationship of $E_{R}$ and $\Delta S(Q, \omega)$ vs $\Delta_{\text {tot }}$. (c) $E_{R}$ vs $T_{c}$ for $\mathrm{CaKFe}_{4} \mathrm{As}_{4}$ single crystal and powder samples under the linear scaling: $E_{R}=4.9 k_{B} T_{c}$. (d) The linear scaling between $\Delta_{\mathrm{tot}}$ and $E_{R}$ for iron-based superconductors [21-31,44,72-86]. The dashed line marks $E_{R}=\Delta_{\text {tot }}$, and the solid line is $E_{R}=0.64 \Delta_{\text {tot }}[7]$. together with our results of $\mathrm{CaKFe}_{4} \mathrm{As}_{4}$. Another wellknown scaling behavior with $E_{R}=4.9 k_{B} T_{c}$ may be still applicable in this new compound [26,40], only if considering the average resonance energy $E_{R}=12.5 \mathrm{meV}$ determined on a powder sample [Fig. 4(c)] [87]. It should be noticed that all pockets are fully gapped in the superconducting state, and there is no evidence for gap modulation along $k_{z}$ or gap nodes in the spectroscopic investigations $[72,88-90]$. This agrees with the nondispersive feature of all three resonant modes and rules out the sign-changed gaps within a single Fermi pocket. Moreover, the orbital selective pairing could generate double resonant modes and possibly even $L$ modulation, as shown in the $\mathrm{NaFe}_{1-x} \mathrm{Co}_{x}$ As system $[25,34,49]$. Unfortunately, further analysis on the orbital selection of pairing in $\mathrm{CaKFe}_{4} \mathrm{As}_{4}$ would be very difficult, given the equal occupation of $\mathrm{Fe}$ orbitals, including $d_{x z}, d_{y z}, d_{x^{2}-y^{2}}$, and $d_{z^{2}}$ [71].

More importantly, the $\mathrm{CaKFe}_{4} \mathrm{As}_{4}$ compound actually is a bilayer system where the two Fe-As layers linked by $\mathrm{Ca}$ have a shorter distance along the $c$ axis than those linked by $\mathrm{K}$ for their different ionic radius [53,54,72,91,92] [Fig. 1(a)], thus the magnetic coupling within the bilayer unit is much stronger than the interbilayer interaction. We also notice that the interlayer exchange coupling $S J_{c}$ in $\mathrm{CaFe}_{2} \mathrm{As}_{2}$ is much larger (about $5.5 \mathrm{meV}$ ) [93] than that in $\mathrm{BaFe}_{2} \mathrm{As}_{2}$ (about $0.22 \mathrm{meV}$ ) [94], and almost zero in $\mathrm{KFe}_{2} \mathrm{As}_{2}[95,96]$, accompanied by the stretched Fe-As interlayer spacing with $0.5 c=5.84,6.51$, and $6.94 \AA$ [97-100], respectively. The distance of the Fe-Fe plane within one $\mathrm{Fe}$-As bilayer of $\mathrm{CaKFe}_{4} \mathrm{As}_{4}$ is $0.4636 c=$ $5.855 \AA$, almost the same as the Fe-As interlayer spacing in $\mathrm{CaFe}_{2} \mathrm{As}_{2}$. Moreover, the energy difference between the odd $(13 \mathrm{meV})$ and even $(18.3 \mathrm{meV})$ spin resonance peaks is $5.3 \mathrm{meV}$, similar to $S J_{c}$ in $\mathrm{CaFe}_{2} \mathrm{As}_{2}$. All these facts closely resemble those in the metallic $\mathrm{YBCO}$ with strong intrabilayer coupling $J_{\perp} \sim 10 \mathrm{meV}$ in the magnetically decoupled bilayers, where two spin resonance modes are found at 41 and $53 \mathrm{meV}$ following the odd and even $L$ symmetries, respectively $[51,70]$. The existence of two spin resonance modes in $\mathrm{YBCO}$ and $\mathrm{Bi} 2212$ indicates that there is still an AFM coupling between $\mathrm{Cu}-\mathrm{O}$ planes even in the superconducting state, which probably drives the bilayer systems to higher $T_{c}$ than the monolayer systems [5,6]; whereas the multiband nature of $\mathrm{CaKFe}_{4} \mathrm{As}_{4}$ induces further splitting of the odd modes, thus generating triple peaks of spin resonance.

It should be noticed that the even mode of spin resonance in cuprates always has weaker intensity and higher energy than the odd mode. This is attributed to the presence of a threshold of the electron-hole spin flip continuum slightly below $2 \Delta$, which supports the spin exciton scenario [6]. Although the dichotomy of theoretical descriptions of magnetism is still an unresolved issue in iron-based superconductors, the nearly isotropic spin resonance in most compounds basically agrees with the spin- 1 exciton picture 
$[2,3,26,40]$. The multiple resonant modes remind us to recall the broadening and asymmetric spin resonance peak in many other iron-based superconducting systems, which are more likely induced by several overlapped odd and even modes due to small $S J_{c}$ [21-32]. If in analogy to the case of $\mathrm{CaKFe}_{4} \mathrm{As}_{4}$, the low-energy part of the resonance peak is probably filled with odd modes, while the even modes mostly contribute to the high-energy part. When changing $L$ from odd to even within one Brillouin zone, the overall peak position will naturally shift to higher energy [56]. This makes the resonant mode in appearance with a dispersion along the $L$ direction [25-34]. Finally, compared with our recent discovery of $2 \mathrm{D}$ spin resonance under threedimensional Fermi surfaces in a 112-type iron-based superconductor [40], it suggests that the resonance intensity is much more sensitive to the local magnetic couplings rather than the $k_{z}$ dependence of fermiology, even though the resonant energy is mostly coupled to superconducting gaps from itinerant electrons near the Fermi surfaces.

In summary, we have discovered strongly $L$-dependent triple spin resonance modes in the new iron-based superconductor $\mathrm{CaKFe}_{4} \mathrm{As}_{4}$ under multiple pairing channels. The resonance energies are below and proportional to the total superconducting gaps, consistent with the $s_{ \pm}$pairing mechanism. Both odd and even $L$ symmetries of the resonance intensity are found, which are attributed to the nondegenerate spin excitations from the Fe-As bilayer similar to the cuprate superconductors with the $\mathrm{Cu}-\mathrm{O}$ bilayer. Our results suggest that the spin resonance in iron-based superconductors has an intrinsic common nature with cuprate superconductors, and the high- $T_{c}$ superconductivity in both families is strongly associated with local magnetic interactions coupled with itinerant electrons.

This work is supported by the National Key Research and Development Program of China (2017YFA0302903, 2017YFA0303103, 2016YFA0300502, 2015CB921300, 2017YFA0303100), the National Natural Science Foundation of China (11374011, 11374346, 11674406, 11334012, and 11674372), the Strategic Priority Research Program (B) of the Chinese Academy of Sciences (CAS) (XDB07020300), and the Key Research Program of the CAS (XDPB01). H. L. is grateful for the support from the Youth Innovation Promotion Association of CAS (2016004). This work is based on experiments performed at the Swiss Spallation Neutron Source (SINQ), Paul Scherrer Institute, Villigen, Switzerland. The neutron experiment at the Materials and Life Science Experimental Facility of J-PARC was performed under a user program (Proposal No. 2017A0051).

*hqluo@iphy.ac.cn

[1] J. M. Tranquada, G. Xu, and I. A. Zaliznyak, J. Magn. Magn. Mater. 350, 148 (2014).

[2] D. S. Inosov, C. R. Phys. 17, 60 (2016).
[3] P. Dai, Rev. Mod. Phys. 87, 855 (2015).

[4] O. Stockert, J. Arndt, E. Faulhaber, C. Geibel, H. S. Jeevan, S. Kirchner, M. Loewenhaupt, K. Schmalzl, W. Schmidt, Q. Si, and F. Steglich, Nat. Phys. 7, 119 (2011).

[5] M. Eschrig, Adv. Phys. 55, 47 (2006).

[6] Y. Sidis, S. Pailhès, V. Hinkov, B. Fauqué, C. Ulrich, L. Capogna, A. Ivanov, L.-P. Regnault, B. Keimer, and P. Bourges, C.R. Phys. 8, 745 (2007).

[7] G. Yu, Y. Li, E. M. Motoyama, and M. Greven, Nat. Phys. 5, 873 (2009).

[8] Q. Si, R. Yu, and E. Abrahams, Nat. Rev. Mater. 1, 16017 (2016).

[9] P. Richard, T. Sato, K. Nakayama, T. Takahashi, and H. Ding, Rep. Prog. Phys. 74, 124512 (2011).

[10] M. M. Korshunov and I. Eremin, Phys. Rev. B 78, 140509(R) (2008).

[11] A. V. Chubukov, D. V. Efremov, and I. Eremin, Phys. Rev. B 78, 134512 (2008).

[12] T. A. Maier, S. Graser, D. J. Scalapino, and P. Hirschfeld, Phys. Rev. B 79, 134520 (2009).

[13] I. Mazin and J. Schmalian, Physica (Amsterdam) 469C, 614 (2009).

[14] K. J. Seo, B. A. Bernevig, and J. Hu, Phys. Rev. Lett. 101, 206404 (2008).

[15] T. Hanaguri, S. Niitaka, K. Kuroki, and H. Takagi, Science 328, 474 (2010).

[16] H. Yang, Z. Wang, D. Fang, Q. Deng, Q. Wang, Y. Xiang, Y. Yang, and H. Wen, Nat. Commun. 4, 2749 (2013).

[17] A. A. Kalenyuk, A. Pagliero, E. A. Borodianskyi, A. A. Kordyuk, and V. M. Krasnov, Phys. Rev. Lett. 120, 067001 (2018).

[18] Z. Du, X. Yang, H. Lin, D. Fang, G. Du, J. Xing, H. Yang, X. Zhu, and H.-H. Wen, Nat. Commun. 7, 10565 (2016).

[19] Z. Du, X. Yang, D. Altenfeld, Q. Gu, H. Yang, I. Eremin, P. J. Hirschfeld, I. I. Mazin, H. Lin, X. Zhu, and H.-H. Wen, Nat. Phys. 14, 134 (2018).

[20] A. D. Christianson, E. A. Goremychkin, R. Osborn, S. Rosenkranz, M. D. Lumsden, C. D. Malliakas, I. S. Todorov, H. Claus, D. Y. Chung, M. G. Kanatzidis, R. I. Bewley, and T. Guidi, Nature (London) 456, 930 (2008).

[21] Y. Qiu, W. Bao, Y. Zhao, C. Broholm, V. Stanev, Z. Tesanovic, Y. C. Gasparovic, S. Chang, J. Hu, B. Qian, M. Fang, and Z. Mao, Phys. Rev. Lett. 103, 067008 (2009).

[22] D. S. Inosov, J. T. Park, P. Bourges, D. L. Sun, Y. Sidis, A. Schneidewind, K. Hradil, D. Haug, C. T. Lin, B. Keimer, and V. Hinkov, Nat. Phys. 6, 178 (2010).

[23] N. Qureshi, P. Steffens, Y. Drees, A. C. Komarek, D. Lamago, Y. Sidis, L. Harnagea, H.-J. Grafe, S. Wurmehl, B. Büchner, and M. Braden, Phys. Rev. Lett. 108, 117001 (2012).

[24] S. Wakimoto, K. Kodama, M. Ishikado, M. Matsuda, R. Kajimoto, M. Arai, K. Kakurai, F. Esaka, A. Iyo, H. Kito, H. Eisaki, and S. Shamoto, J. Phys. Soc. Jpn. 79, 074715 (2010).

[25] C. Zhang, R. Yu, Y. Su, Y. Song, M. Wang, G. Tan, T. Egami, J. A. Fernandez-Baca, E. Faulhaber, Q. Si, and P. Dai, Phys. Rev. Lett. 111, 207002 (2013).

[26] P. D. Johnson, G. Xu, and W.-G. Yin, Iron-Based Superconductivity (Springer, New York, 2015), pp. 165-169. 
[27] M. Wang, H. Luo, J. Zhao, C. Zhang, M. Wang, K. Marty, S. Chi, J. W. Lynn, A. Schneidewind, S. Li, and P. Dai, Phys. Rev. B 81, 174524 (2010).

[28] H. Luo, X. Lu, R. Zhang, M. Wang, E. A. Goremychkin, D. T. Adroja, S. Danilkin, G. Deng, Z. Yamani, and P. Dai, Phys. Rev. B 88, 144516 (2013).

[29] S. Chi, A. Schneidewind, J. Zhao, L. W. Harriger, L. Li, Y. Luo, G. Cao, Z. Xu, M. Loewenhaupt, J. Hu, and P. Dai, Phys. Rev. Lett. 102, 107006 (2009).

[30] C. Zhang, M. Wang, H. Luo, M. Wang, M. Liu, J. Zhao, D. L. Abernathy, T. A. Maier, K. Marty, M. D. Lumsden, S. Chi, S. Chang, J. A. Rodriguez-Rivera, J. W. Lynn, T. Xiang, J. Hu, and P. Dai, Sci. Rep. 1, 115 (2011).

[31] C. H. Lee, P. Steffens, N. Qureshi, M. Nakajima, K. Kihou, A. Iyo, H. Eisaki, and M. Braden, Phys. Rev. Lett. 111, 167002 (2013).

[32] J. Zhao, C. R. Rotundu, K. Marty, M. Matsuda, Y. Zhao, C. Setty, E. Bourret-Courchesne, J. Hu, and R. J. Birgeneau, Phys. Rev. Lett. 110, 147003 (2013).

[33] M. G. Kim, G. S. Tucker, D. K. Pratt, S. Ran, A. Thaler, A. D. Christianson, K. Marty, S. Calder, A. Podlesnyak, S. L. Budko, P. C. Canfield, A. Kreyssig, A. I. Goldman, and R. J. McQueeney, Phys. Rev. Lett. 110, 177002 (2013).

[34] C. Zhang, H.-F. Li, Y. Song, Y. Su, G. Tan, T. Netherton, C. Redding, S. V. Carr, O. Sobolev, A. Schneidewind, E. Faulhaber, L. W. Harriger, S. Li, X. Lu, D. X. Yao, T. Das, A. V. Balatsky, T. Brückel, J. W. Lynn, and P. Dai, Phys. Rev. B 88, 064504 (2013).

[35] J. T. Park, G. Friemel, Y. Li, J.-H. Kim, V. Tsurkan, J. Deisenhofer, H.-A. K. von Nidda, A. Loidl, A. Ivanov, B. Keimer, and D. S. Inosov, Phys. Rev. Lett. 107, 177005 (2011).

[36] G. Friemel, W. P. Liu, E. A. Goremychkin, Y. Liu, J. T. Park, O. Sobolev, C. T. Lin, B. Keimer, and D. S. Inosov, Europhys. Lett. 99, 67004 (2012).

[37] M. A. Surmach, F. Brückner, S. Kamusella, R. Sarkar, P. Y. Portnichenko, J. T. Park, G. Ghambashidze, H. Luetkens, P. K. Biswas, W. J. Choi, Y. I. Seo, Y. S. Kwon, H.-H. Klauss, and D. S. Inosov, Phys. Rev. B 91, 104515 (2015).

[38] Q. Wang, Y. Shen, B. Pan, Y. Hao, M. Ma, F. Zhou, P. Steffens, K. Schmalzl, T. R. Forrest, M. Abdel-Hafiez, X. Chen, D. A. Chareev, A. N. Vasiliev, P. Bourges, Y. Sidis, H. Cao, and J. Zhao, Nat. Mater. 15, 159 (2016).

[39] M. Ma, L. Wang, P. Bourges, Y. Sidis, S. Danilkin, and Y. Li, Phys. Rev. B 95, 100504(R) (2017).

[40] T. Xie, D. Gong, H. Ghosh, A. Ghosh, M. Soda, T. Masuda, S. Itoh, F. Bourdarot, L.-P. Regnault, S. Danilkin, S. Li, and H. Luo, Phys. Rev. Lett. 120, 137001 (2018).

[41] L. W. Harriger, O. J. Lipscombe, C. Zhang, H. Luo, M. Wang, K. Marty, M. D. Lumsden, and P. Dai, Phys. Rev. B 85, 054511 (2012).

[42] S. Onari, H. Kontani, and M. Sato, Phys. Rev. B 81, 060504(R) (2010).

[43] S. Onari and H. Kontani, Phys. Rev. Lett. 109, 137001 (2012).

[44] Q. Wang, J. T. Park, Y. Feng, Y. Shen, Y. Hao, B. Pan, J. W. Lynn, A. Ivanov, S. Chi, M. Matsuda, H. Cao, R. J. Birgeneau, D. V. Efremov, and J. Zhao, Phys. Rev. Lett. 116, 197004 (2016).
[45] H. Luo, M. Wang, C. Zhang, X. Lu, L.-P. Regnault, R. Zhang, S. Li, J. Hu, and P. Dai, Phys. Rev. Lett. 111, 107006 (2013).

[46] P. Steffens, C. H. Lee, N. Qureshi, K. Kihou, A. Iyo, H. Eisaki, and M. Braden, Phys. Rev. Lett. 110, 137001 (2013).

[47] M. Ma, P. Bourges, Y. Sidis, Y. Xu, S. Li, B. Hu, J. Li, F. Wang, and Y. Li, Phys. Rev. X 7, 021025 (2017).

[48] D. Hu, W. Zhang, Y. Wei, B. Roessli, M. Skoulatos, L.-P. Regnault, G. Chen, Y. Song, H. Luo, S. Li, and P. Dai, Phys. Rev. B 96, 180503(R) (2017).

[49] W. Wang, J. T. Park, R. Yu, Y. Li, Y. Song, Z. Zhang, A. Ivanov, J. Kulda, and P. Dai, Phys. Rev. B 95, 094519 (2017).

[50] S. Pailhès, Y. Sidis, P. Bourges, C. Ulrich, V. Hinkov, L.-P. Regnault, A. Ivanov, B. Liang, C. T. Lin, C. Bernhard, and B. Keimer, Phys. Rev. Lett. 91, 237002 (2003).

[51] S. Pailhès, Y. Sidis, P. Bourges, V. Hinkov, A. Ivanov, C. Ulrich, L.-P. Regnault, and B. Keimer, Phys. Rev. Lett. 93, 167001 (2004).

[52] L. Capogna, B. Fauqué, Y. Sidis, C. Ulrich, P. Bourges, S. Pailhès, A. Ivanov, J. L. Tallon, B. Liang, C. T. Lin, A. I. Rykov, and B. Keimer, Phys. Rev. B 75, 060502(R) (2007).

[53] A. Iyo, K. Kawashima, T. Kinjo, T. Nishio, S. Ishida, H. Fujihisa, Y. Gotoh, K. Kihou, H. Eisaki, and Y. Yoshida, J. Am. Chem. Soc. 138, 3410 (2016).

[54] W. R. Meier, T. Kong, U. S. Kaluarachchi, V. Taufour, N. H. Jo, G. Drachuck, A. E. Böhmer, S. M. Saunders, A. Sapkota, A. Kreyssig, M. A. Tanatar, R. Prozorov, A. I. Goldman, F. F. Balakirev, A. Gurevich, S. L. Bud'ko, and P. C. Canfield, Phys. Rev. B 94, 064501 (2016).

[55] W. R. Meier, T. Kong, S. L. Bud'ko, and P. C. Canfield, Phys. Rev. Mater. 1, 013401 (2017).

[56] See Supplemental Material at http://link.aps.org/ supplemental/10.1103/PhysRevLett.120.267003 for the sample characterization and raw data of neutron scattering experiment, which includes Refs. [57-63].

[57] Q. Fan, W. H. Zhang, X. Liu, Y. J. Yan, M. Q. Ren, R. Peng, H. C. Xu, B. P. Xie, J. P. Hu, T. Zhang, and D. L. Feng, Nat. Phys. 11, 946 (2015).

[58] D. Liu et al., Nat. Commun. 3, 931 (2012).

[59] S. He et al., Nat. Mater. 12, 605 (2013).

[60] S. Tan, Y. Zhang, M. Xia, Z. Ye, F. Chen, X. Xie, R. Peng, D. Xu, Q. Fan, H. Xu, J. Jiang, T. Zhang, X. Lai, T. Xiang, J. Hu, B. Xie, and D. Feng, Nat. Mater. 12, 634 (2013).

[61] J. He et al., Proc. Natl. Acad. Sci. USA 111, 18501 (2014).

[62] L. Zhao et al., Nat. Commun. 7, 10608 (2016).

[63] X. Liu et al., Nat. Commun. 5, 5047 (2014).

[64] U. Stuhr, B. Roessli, S. Gvasaliya, H. M. Rønnow, U. Filges, D. Graf, A. Bollhalder, D. Hohl, R. Bürge, M. Schild, L. Holitzner, C. Kaegi, P. Keller, and T. Mühlebach, Nucl. Instrum. Methods Phys. Res., Sect. A 853, 16 (2017).

[65] M. Nakamura, R. Kajimoto, Y. Inamura, F. Mizuno, M. Fujita, T. Yokoo, and M. Arai, J. Phys. Soc. Jpn. 78, 093002 (2009).

[66] R. Kajimoto et al., J. Phys. Soc. Jpn. 80, SB025 (2011).

[67] Y. Inamura, T. Nakatani, J. Suzuki, and T. Otomo, J. Phys. Soc. Jpn. 82, SA031 (2013). 
[68] W. R. Meier, Q.-P. Ding, A. Kreyssig, S. L. Budko, A. Sapkota, K. Kothapalli, V. Borisov, R. Valent, C. D. Batista, P. P. Orth, R. M. Fernandes, A. I. Goldman, Y. Furukawa, A. E. Böhmer, and P. C. Canfield, npj Quantum Mater. 3, 5 (2018).

[69] D. Reznik, P. Bourges, H. F. Fong, L. P. Regnault, J. Bossy, C. Vettier, D. L. Milius, I. A. Aksay, and B. Keimer, Phys. Rev. B 53, R14741(R) (1996).

[70] H. F. Fong, P. Bourges, Y. Sidis, L. P. Regnault, J. Bossy, A. Ivanov, D. L. Milius, I. A. Aksay, and B. Keimer, Phys. Rev. B 61, 14773 (2000).

[71] F. Lochner, F. Ahn, T. Hickel, and Ilya Eremin, Phys. Rev. B 96, 094521 (2017).

[72] D. Mou, T. Kong, W. R. Meier, F. Lochner, L. L. Wang, Q. Lin, Y. Wu, S. L. Budko, I. Eremin, D. D. Johnson, P. C. Canfield, and A. Kaminski, Phys. Rev. Lett. 117, 277001 (2016).

[73] K. Nakayama, T. Sato, P. Richard, Y.-M. Xu, Y. Sekiba, S. Souma, G. F. Chen, J. L. Luo, N. L. Wang, H. Ding, and T. Takahashi, Europhys. Lett. 85, 67002 (2009).

[74] K. Nakayama, T. Sato, P. Richard, Y.-M. Xu, T. Kawahara, K. Umezawa, T. Qian, M. Neupane, G. F. Chen, H. Ding, and T. Takahashi, Phys. Rev. B 83, 020501(R) (2011).

[75] C. H. Lee, K. Kihou, J. T. Park, K. Horigane, K. Fujita, F. Waßer, N. Qureshi, Y. Sidis, J. Akimitsu, and M. Braden, Sci. Rep. 6, 23424 (2016).

[76] K. Terashima, Y. Sekiba, J. H. Bowen, K. Nakayama, T. Kawahara, T. Sato, P. Richard, Y.-M. Xu, L. J. Li, G. H. Cao, Z.-A. Xu, H. Ding, and T. Takahashi, Proc. Natl. Acad. Sci. USA 106, 7330 (2009).

[77] M. Wang, M. Yi, H. L. Sun, P. Valdivia, M. G. Kim, Z. J. Xu, T. Berlijn, A. D. Christianson, S. Chi, M. Hashimoto, D. H. Lu, X. D. Li, E. Bourret-Courchesne, P. Dai, D. H. Lee, T. A. Maier, and R. J. Birgeneau, Phys. Rev. B 93, 205149 (2016).

[78] Y. Zhang, Z. Ye, Q. Ge, F. Chen, J. Jiang, M. Xu, B. Xie, and D. Feng, Nat. Phys. 8, 371 (2012).

[79] J. Maletz, V. B. Zabolotnyy, D. V. Evtushinsky, S. Thirupathaiah, A. U. B. Wolter, L. Harnagea, A. N. Yaresko, A. N. Vasiliev, D. A. Chareev, A. E. Böhmer, F. Hardy, T. Wolf, C. Meingast, E. D. L. Rienks, B. Büchner, and S. V. Borisenko, Phys. Rev. B 89, 220506(R) (2014).

[80] H. Miao, P. Richard, Y. Tanaka, K. Nakayama, T. Qian, K. Umezawa, T. Sato, Y.-M. Xu, Y. B. Shi, N. Xu, X.-P. Wang, P. Zhang, H.-B. Yang, Z.-J. Xu, J. S. Wen, G.-D. Gu, X. Dai, J.-P. Hu, T. Takahashi, and H. Ding, Phys. Rev. B 85, 094506 (2012).

[81] Q. Q. Ge, Z. R. Ye, M. Xu, Y. Zhang, J. Jiang, B. P. Xie, Y. Song, C. L. Zhang, P. Dai, and D. L. Feng, Phys. Rev. X 3, 011020 (2013).

[82] Z.-S. Wang, Z.-Y. Wang, H.-Q. Luo, X.-Y. Lu, J. Zhu, C.-H. Li, L. Shan, H. Yang, H.-H. Wen, and C. Ren, Phys. Rev. B 86, 060508(R) (2012).
[83] Y. V. Pustovit and A. A. Kordyuk, J. Low Temp. Phys. 42, 995 (2016).

[84] J. Zhu, Z. Wang, Z. Wang, X. Hou, H. Luo, X. Lu, C. Li, L. Shan, H. Wen, and C. Ren, Chin. Phys. Lett. 32, 077401 (2015).

[85] Y. Zhang, L. X. Yang, M. Xu, Z. R. Ye, F. Chen, C. He, H. C. Xu, J. Jiang, B. P. Xie, J. J. Ying, X. F. Wang, X. H. Chen, J. P. Hu, M. Matsunami, S. Kimura, and D. L. Feng, Nat. Mater. 10, 273 (2011).

[86] X. H. Niu, S. D. Chen, J. Jiang, Z. R. Ye, T. L. Yu, D. F. Xu, M. Xu, Y. Feng, Y. J. Yan, B. P. Xie, J. Zhao, D. C. Gu, L. L. Sun, Q. Mao, H. Wang, M. Fang, C. J. Zhang, J. P. $\mathrm{Hu}$, Z. Sun, and D. L. Feng, Phys. Rev. B 93, 054516 (2016).

[87] K. Iida, M. Ishikado, Y. Nagai, H. Yoshida, A. D. Christianson, N. Murai, K. Kawashima, Y. Yoshida, H. Eisaki, and A. Iyo, J. Phys. Soc. Jpn. 86, 093703 (2017).

[88] R. Yang, Y. Dai, B. Xu, W. Zhang, Z. Qiu, Q. Sui, C. C. Homes, and X. Qiu, Phys. Rev. B 95, 064506 (2017).

[89] P. K. Biswas, A. Iyo, Y. Yoshida, H. Eisaki, K. Kawashima, and A. D. Hillier, Phys. Rev. B 95, 140505(R) (2017).

[90] K. Cho, A. Fente, S. Teknowijoyo, M. A. Tanatar, K. R. Joshi, N. M. Nusran, T. Kong, W. R. Meier, U. Kaluarachchi, I. Guillamón, H. Suderow, S. L. Bud'ko, P. C. Canfield, and R. Prozorov, Phys. Rev. B 95, 100502(R) (2017).

[91] J. Cui, Q.-P. Ding, W. R. Meier, A. E. Böhmer, T. Kong, V. Borisov, Y. Lee, S. L. Bud'ko, R. Valentí, P. C. Canfield, and Y. Furukawa, Phys. Rev. B 96, 104512 (2017).

[92] Q.-P. Ding, W. R. Meier, A. E. Böhmer, S. L. Bud'ko, P. C. Canfield, and Y. Furukawa, Phys. Rev. B 96, 220510(R) (2017).

[93] J. Zhao, D. T. Adroja, D.-X. Yao, R. Bewley, S. Li, X. F. Wang, G. Wu, X. H. Chen, J. Hu, and P. Dai, Nat. Phys. 5, 555 (2009).

[94] J. T. Park, G. Friemel, T. Loew, V. Hinkov, Y. Li, B. H. Min, D. L. Sun, A. Ivanov, A. Piovano, C. T. Lin, B. Keimer, Y. S. Kwon, and D. S. Inosov, Phys. Rev. B 86, 024437 (2012).

[95] K. Horigane, K. Kihou, K. Fujita, R. Kajimoto, K. Ikeuchi, S. Ji, J. Akimitsu, and C. H. Lee, Sci. Rep. 6, 33303 (2016).

[96] M. Wang, C. Zhang, X. Lu, G. Tan, H. Luo, Y. Song, M. Wang, X. Zhang, E. A. Goremychkin, T. G. Perring, T. A. Maier, Z. Yin, K. Haule, G. Kotliar, and P. Dai, Nat. Commun. 4, 2874 (2013).

[97] N. Ni, S. Nandi, A. Kreyssig, A. I. Goldman, E. D. Mun, S. L. Bud'ko, and P. C. Canfield, Phys. Rev. B 78, 014523 (2008).

[98] M. Rotter, M. Tegel, D. Johrendt, I. Schellenberg, W. Hermes, and R. Pöttgen, Phys. Rev. B 78, 020503(R) (2008).

[99] H. Luo, Z. Wang, H. Yang, P. Cheng, X. Zhu, and H.-H. Wen, Supercond. Sci. Technol. 21, 125014 (2008).

[100] K. Kihou, T. Saito, S. Ishida, M. Nakajima, Y. Tomioka, H. Fukazawa, Y. Kohori, T. Ito, S. Uchida, A. Iyo, C.-H. Lee, and H. Eisaki, J. Phys. Soc. Jpn. 79, 124713 (2010). 